\title{
Prevalence and progression of visual impairment in patients newly diagnosed with clinical type 2 diabetes: a 6-year follow up study
}

\author{
Niels de Fine Olivarius ${ }^{1 *}$, Volkert Siersma ${ }^{1}$, Gitte Juul Almind ${ }^{1}$, Niels Vesti Nielsen ${ }^{2}$
}

\begin{abstract}
Background: Many diabetic patients fear visual loss as the worst consequence of diabetes. In most studies the main eye pathology is assigned as the cause of visual impairment. This study analysed a broad range of possible ocular and non-ocular predictors of visual impairment prospectively in patients newly diagnosed with clinical type 2 diabetes.

Methods: Data were from a population-based cohort of 1,241 persons newly diagnosed with clinical, often symptomatic type 2 diabetes aged $\geq 40$ years. After 6 years, 807 patients were followed up. Standard eye examinations were done by practising ophthalmologists.
\end{abstract}

Results: At diabetes diagnosis median age was 65.5 years. Over 6 years, the prevalence of blindness (visual acuity of best seeing eye $\leq 0.1)$ rose from $0.9 \%(11 / 1,241)$ to $2.4 \%(19 / 807)$ and the prevalence of moderate visual impairment $(>0.1 ;<0.5)$ rose from $5.4 \%(67 / 1,241)$ to $6.7 \%$ (54/807). The incidence (95\% confidence interval) of blindness was 40.2 (25.3-63.8) per 10,000 patient-years. Baseline predictors of level of visual acuity (age, age-related macular degeneration (AMD), cataract, living alone, low self-rated health, and sedentary life-style) and speed of continued visual loss (age, AMD, diabetic retinopathy (DR), cataract, living alone, and high fasting triglycerides) were identified.

Conclusions: In a comprehensive assessment of predictors of visual impairment, even in a health care system allowing self-referral to free eye examinations, treatable eye pathologies such as DR and cataract emerge together with age as the most notable predictors of continued visual loss after diabetes diagnosis. Our results underline the importance of eliminating barriers to efficient eye care by increasing patients' and primary care practitioners' awareness of the necessity of regular eye examinations and timely surgical treatment.

\section{Background}

In Europe and the United States severe visual impairment may be 2-3 times more common among people with diabetes than in the general population $[1,2]$, but this difference decreases with age [2]. Diabetic retinopathy (DR) is regarded as the cause of blindness in 5-15\% of the blind in the general population [3-5] and in $30-50 \%$ of blind type 2 diabetic patients [6,7]. There are, however, huge regional differences in presumed causes of blindness across the world [8]. DR is considered the

\footnotetext{
* Correspondence: olivarius@sund.ku.dk

'The Research Unit for General Practice and Section of General Practice, Department of Public Health, University of Copenhagen, Copenhagen, Denmark

Full list of author information is available at the end of the article
}

leading cause of blindness among people of working age in many countries [3-5], while age-related macular degeneration (AMD) is considered the leading cause in people over 65 years [3-5,9].

Diabetes with even mildly to moderately impaired sight has a negative impact on perceived quality of life and psychosocial functioning giving rise to feelings of vulnerability, worries about the future and loss of independence and mobility [10-12]. A sizeable proportion of type 2 diabetic patients fear visual loss intensely [13] and consider loss of vision the worst complication of diabetes [10]. For the health practitioner visual acuity is a ubiquitous and handy measure of visual function, but visual acuity is not a suitable measure of future visual loss as the sight-threatening eye pathologies often are

\section{Ciomed Central}


present for many years before vision begins to decline as a result of these pathologies.

In the history of diabetes treatment, the development of diabetic retinopathy has been included in the outcome of numerous clinical trials though prevention of visual loss is the ultimate target for the patients. While the ocular predictors of future visual loss in diabetic patients are well-described [14-19], a comprehensive prospective study of ocular and non-ocular predictors of long-term changes in measured visual acuity has not been published before [14,16,19-23].

Our main aim was to study a broad range of predictors of vision loss in a population-based sample of patients newly diagnosed with type 2 diabetes and observed for 6 years.

\section{Methods}

\section{Study population}

In the Danish Diabetes Care in General Practice study [24], 474 general practitioners agreed to include all subjects with newly diagnosed diabetes on their practice list (Figure 1). The 140 patients without a measurement of visual acuity at diagnosis did not differ from the 1,241 patients who were included in the present study regarding age $(p=0.36)$, sex $(p=0.31)$ and diagnostic plasma glucose $(p=0.81)$. At 6 -year follow up, the 159 noncensored patients without information about visual acuity (Figure 1) did not differ from the 807 re-examined patients with regard to age $(p=0.23)$, sex $(p=$ $0.82)$, diagnostic plasma glucose $(p=0.43)$, prevalence of DR at diagnosis $(3.2 \%(5 / 158)$ vs. $4.4 \%(35 / 800), p=$ 0.49 ) and prevalence of moderately impaired vision or worse at diagnosis (7.0\% (11/159) vs. 3.3\% (27/807), $p=$ $0.10)$. A small number started insulin treatment within 180 days of diagnosis, so $97.6 \%$ of the 1381 patients who had started in the study were considered to have type 2 diabetes [24]. Informed consent was obtained from all participants, and the protocol was approved by the ethics committee for Copenhagen and Frederiksberg.

\section{Ophthalmologic examination}

Immediately after diabetes diagnosis, the general practitioner referred the patient to a practising ophthalmologist who did a standard eye examination. The result of the funduscopy was recorded by the ophthalmologist in a multiple choice question with six response categories: no diabetic lesions, microaneurysms only, background retinopathy without or with maculopathy, and proliferative retinopathy without or with new vessels on the optic disc. In open-ended questions information about other retinal pathologies, glaucoma, and eye operations was given, and the presence of cataract was indicated in a closed question. Information about glaucoma was also given as answers to open-ended questions about reasons
Inclusion criteria:

474 doctors were to include all patients on their practice list fulfilling the following criteria:

1) Diabetes mellitus diagnosed from 1 March 1989 to 28 February 1991 (71 doctors volunteered for a 1-year extension of this period)

2) Based on hyperglycaemic symptoms and/or raised blood glucose values measured in general practice, the diagnosis was established by a single whole blood or plasma glucose concentration $\geq 7.0 / 8.0$ $\mathrm{mmol} / \mathrm{l}$ measured at a major laboratory

3) Age 40 years or older at diagnosis

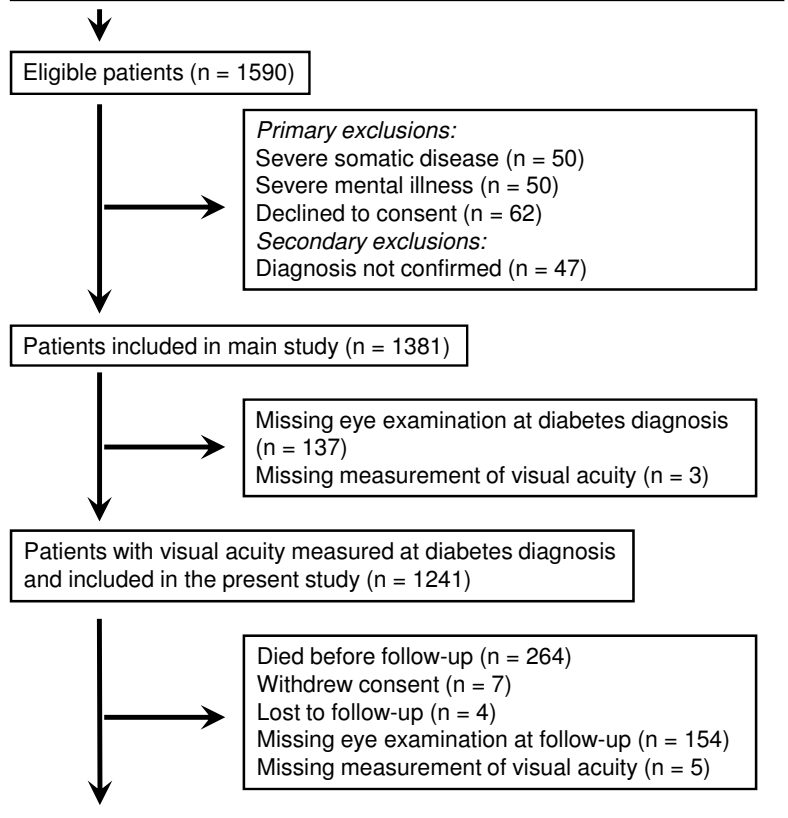

Patients with visual acuity measured at diagnosis and 6-year follow-up $(n=807)$

Figure 1 Flow of participants through study

for impaired vision. Visual acuity with best correction was estimated with an optotype chart, usually a Snellen chart, and given for each eye as the Snellen ratio at 6 metres or 20 feet or as decimal acuity, but all measurements were converted to decimal acuity and logMAR [25]. The time (interquartile range, IQR) from diabetes diagnosis until the first eye examination was 45 (24-83) days. A total of 164 ophthalmologists participated, and $68.5 \%(553 / 807)$ of patients were seen by the same ophthalmologist at first and last eye examination. The eye with best visual acuity was used for evaluation of retinal pathology.

\section{Assessments}

The doctors recorded the following information about the patient: height and weight without shoes and outer garments; blood pressure and heart rate by routine methods after a 10-minute rest in a sitting position; sense of touch of cotton wool and pin prick on both feet; presence of dorsalis pedis or posterior tibial pulse on both feet; presence of patellar reflexes; history of 
myocardial infarction and/or stroke causing hospitalization; and amputation of any part of leg or foot before or at the time of diabetes diagnosis.

In questionnaires, patients gave information about whether they lived alone, their education, familial disposition to diabetes, smoking habits, leisure time physical activity, angina pectoris, intermittent claudication, and global self-rated health.

\section{Definitions}

The decimal acuity of patients' best seeing eye was used to categorise patients according to usual clinical and administrative practice in Denmark: normal vision $(\geq 0.5)$, moderate visual impairment $(<0.5$ and $>0.1)$ or blindness $(\leq 0.1)$. Cardiovascular disease was defined as history of myocardial infarction and/or history of stroke and/or angina pectoris and/or intermittent claudication and/or absent arterial pulses on both feet and/or amputation on the lower extremities. Peripheral neuropathy was defined as lack of a sense of pin prick and/or touch of cotton wool on at least one foot and/or absent patellar reflex on at least one knee.

\section{Assays}

Laboratory analyses were centralized and quality controlled. Fasting blood samples were analysed at Odense University Hospital. Throughout the study, haemoglobin $\mathrm{A} 1 \mathrm{c}\left(\mathrm{HbA}_{1 \mathrm{c}}\right)$ was determined by the same ion-exchange, high-performance liquid chromatography method. Samples from 100 blood donors (age 20-80 years, 33 men, 67 women) were analysed, and the reference interval (mean $\pm 2 \mathrm{SD}$ ) was calculated to be 5.4-7.4\%. Quality assurance was obtained with commercial control preparations from Bio-Rad. In October-December 1995, the mean (SD) of low $(\mathrm{n}=24)$ and high $(\mathrm{n}=29)$ control samples were $6.7(0.31) \%$ and $10.4(0.63) \%$, respectively, resulting in coefficients of variation $(C V=S D \times 100 /$ mean) of $4.6 \%$ and $6.0 \%$. Serum total cholesterols were measured enzymatically with cholesterol esterasecholesterol oxidase-peroxidase reagent, and fasting serum triglycerides was determined enzymatically with a lipase-glycerolkinase-glycerol-3-phosphate oxidaseperoxidase reagent. Serum creatinine was determined by the Jaffe reaction. In this nationwide study a freshly voided morning urine sample was used instead of timed urine collection to determine renal involvement. Urinary albumin concentration was measured at Århus University Hospital by a polyethylene glycol radioimmunoassay. Spot urinary albumin concentration is of value in predicting both progression of renal involvement and increased mortality, as is albumin excretion rate [26].

\section{Statistical analysis}

The influence of baseline characteristics on change in visual acuity over 6 years was investigated in linear mixed models [27] using all available measurements of visual acuity from the best seeing eye. For each of the characteristics two models were constructed: one model where the change in visual acuity was described by a linear time trend (intercept and slope for the average change in visual acuity during the study) that differed for each of the categories of the characteristic, and a second model where the time trend additionally depended on age and sex in a multivariable regression fashion. Within-patient correlation of the observations was modelled by a patient random effect. The effect of the characteristic on changes in visual acuity was summarized with the fixed effect intercept and slope for each of the covariate classes from the first model (i.e. excluding age and sex). Differences between the covariate categories in intercept and slope in the two models were tested with the appropriate F-tests. In the $5.8 \%$ (72/1241) of patients who had an eye operation in the best seeing eye during follow up, measurements of visual acuity made after the operation were excluded from the analyses.

For the patients who were not moderately visually impaired or blind at baseline, incidence rates for these conditions were estimated as the ratio between (1) the number of patients with moderately impaired vision/ blindness at follow up and (2) the sum of follow up times. The follow up times were halved for patients who became moderately visually impaired or blind during follow up, and confidence intervals were calculated assuming Poisson-distributed occurrences of vision impairment. Simple comparisons were made with Kruskal-Wallis tests and $\chi^{2}$-tests. To account for multiple statistical testing, we applied the method of BenjaminiHochberg [28] on all the four columns of p-values in Table 1, i.e. $4 \times 28=112 \mathrm{p}$-values, and found that a significance level of 0.0179 was to be used in order to control the false discovery rate at 5\%. Accordingly, the nominal level of statistical significance was chosen to be $p<0.05$, except in the mixed models in Table 1 , where it was $p<0.01$.

\section{Results}

Baseline characteristics

At diabetes diagnosis median (IQR) age was 65.5 (56.073.6) years, male/female ratio was 1.11 (653/588), and median diagnostic plasma glucose was 13.7 (10.7-17.0) $\mathrm{mmol} / \mathrm{l}$. The prevalence (95\% confidence interval) of blindness and moderate visual impairment was 0.9 (0.41.4) $\%$ and 5.4 (4.1-6.7) \%, respectively (Table 2 ). AMD, other non-diabetic retinopathy and, above all, cataract were common. The ophthalmologists estimated that cataract was the most common cause of visual impairment except among the blind (Table 2). At diagnosis the eye doctors reported glaucoma in the best seeing eye in 18 
Table 1 Average changes in visual acuity during the first 6 years after diabetes diagnosis according to baseline patient characteristics

\begin{tabular}{|c|c|c|c|c|c|c|c|c|c|c|}
\hline \multirow[b]{2}{*}{$\begin{array}{l}\text { Characteristic } \\
\text { at diagnosis }\end{array}$} & \multirow[b]{2}{*}{ Category } & \multirow[b]{2}{*}{$n$} & \multirow[b]{2}{*}{$\begin{array}{l}\text { Average } \\
\text { logMAR } \\
\text { at } \\
\text { diabetes } \\
\text { diagnosis }\end{array}$} & \multirow[b]{2}{*}{$\begin{array}{l}\text { Average } \\
\text { yearly } \\
\text { change in } \\
\text { logMAR } \\
\text { after } \\
\text { diabetes } \\
\text { diagnosis }\end{array}$} & \multicolumn{2}{|c|}{$\begin{array}{l}\text { Test of difference } \\
\text { in level of logMAR } \\
\text { at diabetes } \\
\text { diagnosis }^{\mathrm{a}}\end{array}$} & \multicolumn{2}{|c|}{$\begin{array}{l}\text { Test of difference } \\
\text { in change of } \\
\text { logMAR after } \\
\text { diabetes } \\
\text { diagnosis }^{\mathrm{b}}\end{array}$} & \multirow[b]{2}{*}{$\begin{array}{l}\text { Median } \\
\text { decimal acuity } \\
\text { at diabetes } \\
\text { diagnosis }\end{array}$} & \multirow[b]{2}{*}{$\begin{array}{c}\text { Median } \\
\text { decimal acuity } \\
5.6 \text { years after } \\
\text { diabetes } \\
\text { diagnosis }\end{array}$} \\
\hline & & & & & $\begin{array}{c}\text { Un- } \\
\text { adjusted } \\
p\end{array}$ & $\begin{array}{l}\text { Age and } \\
\text { sex } \\
\text { adjusted } \\
p\end{array}$ & $\begin{array}{c}\text { Un- } \\
\text { adjusted } \\
p\end{array}$ & $\begin{array}{l}\text { Age and } \\
\text { sex } \\
\text { adjusted } \\
p\end{array}$ & & \\
\hline Total & & 1241 & -0.24 & -0.0250 & & & & & $1.00(0.80-1.00)$ & $0.80(0.67-1.00)$ \\
\hline \multirow[t]{2}{*}{ Sex } & Female & 588 & -0.30 & -0.0343 & 0.001 & 0.070 & 0.0006 & 0.017 & $0.90(0.67-1.00)$ & $0.80(0.60-1.00)$ \\
\hline & Male & 653 & -0.19 & -0.0160 & & & & & $1.00(0.80-1.00)$ & $1.00(0.67-1.00)$ \\
\hline \multirow[t]{3}{*}{ Age (years) } & $40-<60$ & 430 & -0.05 & -0.0068 & $<0.0001$ & $<0.0001$ & $<0.0001$ & $<0.0001$ & $1.00(1.00-1.00)$ & $1.00(0.80-1.00)$ \\
\hline & $60-<70$ & 377 & -0.18 & -0.0125 & & & & & $1.00(0.80-1.00)$ & $0.80(0.67-1.00)$ \\
\hline & $\geq 70$ & 434 & -0.47 & -0.0769 & & & & & $0.70(0.60-0.95)$ & $0.60(0.40-0.80)$ \\
\hline \multirow[t]{2}{*}{ Living alone } & Yes & 384 & -0.39 & -0.0527 & $<0.0001$ & 0.0009 & $<0.0001$ & 0.002 & $0.80(0.67-1.00)$ & $0.67(0.50-0.90)$ \\
\hline & No & 835 & -0.17 & -0.0158 & & & & & $1.00(0.80-1.00)$ & $1.00(0.70-1.00)$ \\
\hline \multirow[t]{2}{*}{ Education } & Basic & 935 & -0.26 & -0.0293 & 0.006 & 0.67 & 0.008 & 0.30 & $1.00(0.70-1.00)$ & $0.80(0.60-1.00)$ \\
\hline & Higher & 252 & -0.15 & -0.0114 & & & & & $1.00(0.90-1.00)$ & $1.00(0.70-1.00)$ \\
\hline \multirow[t]{3}{*}{ Residence } & Countryside & 486 & -0.26 & -0.0269 & 0.41 & 0.98 & 0.56 & 0.60 & $1.00(0.80-1.00)$ & $0.80(0.63-1.00)$ \\
\hline & Larger towns & 430 & -0.24 & -0.0211 & & & & & $1.00(0.80-1.00)$ & $0.80(0.63-1.00)$ \\
\hline & $\begin{array}{l}\text { Copenhagen } \\
\text { area }\end{array}$ & 273 & -0.20 & -0.0211 & & & & & $1.00(0.80-1.00)$ & $0.80(0.67-1.00)$ \\
\hline \multirow{2}{*}{$\begin{array}{l}\text { Familial } \\
\text { disposition to } \\
\text { DM }\end{array}$} & No & 659 & -0.25 & -0.0314 & 0.58 & 0.76 & 0.041 & 0.028 & $1.00(0.75-1.00)$ & $0.80(0.63-1.00)$ \\
\hline & Yes & 466 & -0.23 & -0.0195 & & & & & $1.00(0.80-1.00)$ & $0.90(0.67-1.00)$ \\
\hline \multirow{3}{*}{$\begin{array}{l}\text { Diagnostic } \\
\text { plasma } \\
\text { glucose } \\
(\mathrm{mmol} / \mathrm{l})\end{array}$} & $<10$ & 220 & -0.23 & -0.0227 & 0.93 & 0.47 & 0.36 & 0.063 & $1.00(0.80-1.00)$ & $0.80(0.63-1.00)$ \\
\hline & $10-<17$ & 706 & -0.24 & -0.0281 & & & & & $1.00(0.80-1.00)$ & $0.80(0.67-1.00)$ \\
\hline & $\geq 17$ & 315 & -0.24 & -0.0193 & & & & & $1.00(0.80-1.00)$ & $0.80(0.60-1.00)$ \\
\hline \multirow{3}{*}{$\begin{array}{l}\text { Haemoglobin } \\
A_{1 c}(\%)^{c}\end{array}$} & $<9$ & 298 & -0.20 & -0.0239 & 0.45 & 0.35 & 0.083 & 0.071 & $1.00(0.80-1.00)$ & $0.80(0.67-1.00)$ \\
\hline & $9-<11$ & 347 & -0.24 & -0.0347 & & & & & $1.00(0.80-1.00)$ & $0.90(0.67-1.00)$ \\
\hline & $\geq 11$ & 396 & -0.25 & -0.0195 & & & & & $1.00(0.70-1.00)$ & $0.80(0.60-1.00)$ \\
\hline \multirow{3}{*}{$\begin{array}{l}\text { Total } \\
\text { cholesterol } \\
(\mathrm{mmol} / \mathrm{l})\end{array}$} & $<6$ & 495 & -0.27 & -0.0190 & 0.22 & 0.55 & 0.20 & 0.076 & $1.00(0.70-1.00)$ & $0.80(0.60-1.00)$ \\
\hline & $6-<7$ & 366 & -0.24 & -0.0297 & & & & & $1.00(0.80-1.00)$ & $0.90(0.67-1.00)$ \\
\hline & $\geq 7$ & 358 & -0.20 & -0.0277 & & & & & $1.00(0.80-1.00)$ & $0.80(0.67-1.00)$ \\
\hline
\end{tabular}


Table 1 Average changes in visual acuity during the first 6 years after diabetes diagnosis according to baseline patient characteristics (Continued)

\begin{tabular}{|c|c|c|c|c|c|c|c|c|c|c|}
\hline \multirow{3}{*}{$\begin{array}{l}\text { Fasting } \\
\text { triglycerides } \\
(\mathrm{mmol} / \mathrm{l})\end{array}$} & $<2$ & 616 & -0.24 & -0.0201 & 0.89 & 0.75 & 0.022 & 0.0008 & $1.00(0.80-1.00)$ & $0.80(0.67-1.00)$ \\
\hline & $2-<3$ & 320 & -0.25 & -0.0227 & & & & & $1.00(0.80-1.00)$ & $0.80(0.60-1.00)$ \\
\hline & $\geq 3$ & 279 & -0.23 & -0.0388 & & & & & $1.00(0.80-1.00)$ & $0.80(0.67-1.00)$ \\
\hline \multirow{3}{*}{$\begin{array}{l}\text { Urinary } \\
\text { albumin (mg/l) }\end{array}$} & $<15$ & 703 & -0.19 & -0.0205 & 0.004 & 0.015 & 0.038 & 0.13 & $1.00(0.80-1.00)$ & $0.90(0.67-1.00)$ \\
\hline & $15-<200$ & 437 & -0.31 & -0.0347 & & & & & $1.00(0.70-1.00)$ & $0.80(0.60-1.00)$ \\
\hline & $\geq 200$ & 59 & -0.21 & -0.0172 & & & & & $1.00(0.80-1.00)$ & $0.80(0.60-1.00)$ \\
\hline \multirow{2}{*}{$\begin{array}{l}\text { Serum } \\
\text { creatinine } \\
(\mu \mathrm{mol} / \mathrm{l})\end{array}$} & $<130$ & 1166 & -0.23 & -0.0238 & 0.012 & 0.33 & 0.012 & 0.062 & $1.00(0.80-1.00)$ & $0.80(0.67-1.00)$ \\
\hline & $\geq 130$ & 54 & -0.43 & -0.0634 & & & & & $0.80(0.67-1.00)$ & $0.75(0.50-1.00)$ \\
\hline \multirow{3}{*}{$\begin{array}{l}\text { Height - men } \\
(\mathrm{cm})\end{array}$} & $<165$ & 69 & -0.30 & -0.0273 & 0.11 & 0.58 & 0.53 & 0.96 & $0.95(0.70-1.00)$ & $0.80(0.60-1.00)$ \\
\hline & $165-<180$ & 470 & -0.18 & -0.0156 & & & & & $1.00(0.80-1.00)$ & $1.00(0.67-1.00)$ \\
\hline & $\geq 180$ & 113 & -0.14 & -0.0135 & & & & & $1.00(0.90-1.00)$ & $1.00(0.80-1.00)$ \\
\hline \multirow{3}{*}{$\begin{array}{l}\text { Height - } \\
\text { women }(\mathrm{cm})\end{array}$} & $<150$ & 19 & -0.47 & -0.0587 & 0.050 & 0.47 & 0.12 & 0.91 & $0.70(0.70-0.80)$ & $0.60(0.50-0.80)$ \\
\hline & $150-<165$ & 415 & -0.32 & -0.0390 & & & & & $0.80(0.67-1.00)$ & $0.80(0.50-1.00)$ \\
\hline & $\geq 165$ & 154 & -0.20 & -0.0203 & & & & & $1.00(0.80-1.00)$ & $0.90(0.70-1.00)$ \\
\hline \multirow{3}{*}{$\begin{array}{l}\text { Systolic blood } \\
\text { pressure (mm } \\
\mathrm{Hg})\end{array}$} & $<140$ & 366 & -0.18 & -0.0174 & 0.023 & 0.78 & 0.036 & 0.63 & $1.00(0.80-1.00)$ & $1.00(0.67-1.00)$ \\
\hline & $140-<160$ & 414 & -0.25 & -0.0223 & & & & & $1.00(0.70-1.00)$ & $0.80(0.63-1.00)$ \\
\hline & $\geq 160$ & 459 & -0.28 & -0.0338 & & & & & $1.00(0.75-1.00)$ & $0.80(0.62-1.00)$ \\
\hline \multirow{3}{*}{$\begin{array}{l}\text { Resting heart } \\
\text { rate (bpm) }\end{array}$} & $<80$ & 754 & -0.23 & -0.0208 & 0.17 & 0.09 & 0.040 & 0.066 & $1.00(0.80-1.00)$ & $0.80(0.67-1.00)$ \\
\hline & $80-<90$ & 333 & -0.23 & -0.0361 & & & & & $1.00(0.70-1.00)$ & $0.80(0.60-1.00)$ \\
\hline & $\geq 90$ & 149 & -0.32 & -0.0219 & & & & & $1.00(0.67-1.00)$ & $0.80(0.63-1.00)$ \\
\hline \multirow{2}{*}{$\begin{array}{l}\text { Cardiovascular } \\
\text { disease }\end{array}$} & No & 849 & -0.20 & -0.0224 & $<0.0001$ & 0.045 & 0.035 & 0.55 & $1.00(0.80-1.00)$ & $0.90(0.67-1.00)$ \\
\hline & Yes & 367 & -0.34 & -0.0357 & & & & & $0.90(0.67-1.00)$ & $0.80(0.50-1.00)$ \\
\hline \multirow{2}{*}{$\begin{array}{l}\text { Peripheral } \\
\text { neuropathy }\end{array}$} & No & 992 & -0.22 & -0.0218 & 0.013 & 0.14 & 0.013 & 0.016 & $1.00(0.80-1.00)$ & $0.90(0.67-1.00)$ \\
\hline & Yes & 232 & -0.32 & -0.0392 & & & & & $0.90(0.67-1.00)$ & $0.80(0.60-1.00)$ \\
\hline \multirow[t]{2}{*}{$\begin{array}{l}\text { Self-rated } \\
\text { health }\end{array}$} & $\begin{array}{l}\text { Excellent or } \\
\text { good }\end{array}$ & 557 & -0.18 & -0.0200 & 0.0006 & 0.003 & 0.043 & 0.13 & $1.00(0.80-1.00)$ & $0.90(0.70-1.00)$ \\
\hline & $\begin{array}{l}\text { Fair, poor or } \\
\text { very poor }\end{array}$ & 663 & -0.29 & -0.0309 & & & & & $0.90(0.70-1.00)$ & $0.80(0.60-1.00)$ \\
\hline \multirow[t]{3}{*}{ Smoking } & Never & 372 & -0.33 & -0.0369 & 0.0007 & 0.32 & 0.009 & 0.50 & $0.90(0.67-1.00)$ & $0.80(0.60-1.00)$ \\
\hline & Former & 431 & -0.22 & -0.0206 & & & & & $1.00(0.80-1.00)$ & $0.80(0.63-1.00)$ \\
\hline & Current & 414 & -0.18 & -0.0186 & & & & & $1.00(0.80-1.00)$ & $1.00(0.70-1.00)$ \\
\hline
\end{tabular}


Table 1 Average changes in visual acuity during the first 6 years after diabetes diagnosis according to baseline patient characteristics (Continued)

\begin{tabular}{|c|c|c|c|c|c|c|c|c|c|c|}
\hline \multirow{2}{*}{$\begin{array}{l}\text { Physical } \\
\text { activity }\end{array}$} & Sedentary & 323 & -0.39 & -0.0364 & $<0.0001$ & $<0.0001$ & 0.035 & 0.20 & $0.80(0.60-1.00)$ & $0.70(0.50-1.00)$ \\
\hline & Non-sedentary & 893 & -0.19 & -0.0227 & & & & & $1.00(0.80-1.00)$ & $0.90(0.67-1.00)$ \\
\hline \multirow{3}{*}{$\begin{array}{l}\text { Weight - men } \\
(\mathrm{kg})\end{array}$} & $<80$ & 200 & -0.24 & -0.0172 & 0.19 & 0.85 & 0.64 & 0.81 & $1.00(0.80-1.00)$ & $0.80(0.67-1.00)$ \\
\hline & $80-<90$ & 196 & -0.18 & -0.0204 & & & & & $1.00(0.80-1.00)$ & $1.00(0.80-1.00)$ \\
\hline & $\geq 90$ & 255 & -0.16 & -0.0130 & & & & & $1.00(0.80-1.00)$ & $1.00(0.70-1.00)$ \\
\hline \multirow{3}{*}{$\begin{array}{l}\text { Weight - } \\
\text { women (kg) }\end{array}$} & $<70$ & 200 & -0.39 & -0.0485 & 0.008 & 0.21 & 0.040 & 0.068 & $0.80(0.67-1.00)$ & $0.70(0.50-1.00)$ \\
\hline & $70-<80$ & 157 & -0.29 & -0.0208 & & & & & $0.90(0.67-1.00)$ & $0.80(0.60-1.00)$ \\
\hline & $\geq 80$ & 231 & -0.21 & -0.0319 & & & & & $1.00(0.80-1.00)$ & $0.80(0.63-1.00)$ \\
\hline \multirow{3}{*}{$\begin{array}{l}\text { Diabetic } \\
\text { retinopathy }\end{array}$} & None & 1186 & -0.23 & -0.0243 & 0.040 & 0.012 & $<0.0001$ & 0.0005 & $1.00(0.80-1.00)$ & $0.80(0.67-1.00)$ \\
\hline & $\begin{array}{l}\text { Microaneurysms } \\
\text { only }\end{array}$ & 14 & -0.54 & 0.0174 & & & & & $1.00(0.90-1.00)$ & $0.85(0.80-1.00)$ \\
\hline & $\begin{array}{l}\text { Further } \\
\text { retinopathy }\end{array}$ & 38 & -0.38 & -0.1164 & & & & & $0.80(0.63-1.00)$ & $0.60(0.50-0.80)$ \\
\hline \multirow{2}{*}{$\begin{array}{l}\text { Age-related } \\
\text { macular } \\
\text { degeneration }\end{array}$} & No & 1087 & -0.18 & -0.0170 & $<0.0001$ & $<0.0001$ & $<0.0001$ & $<0.0001$ & $1.00(0.80-1.00)$ & $0.90(0.67-1.00)$ \\
\hline & Yes & 154 & -0.67 & -0.1000 & & & & & $0.67(0.50-0.80)$ & $0.50(0.40-0.67)$ \\
\hline \multirow[t]{2}{*}{ Cataract } & No & 889 & -0.14 & -0.0145 & $<0.0001$ & $<0.0001$ & $<0.0001$ & 0.0004 & $1.00(0.80-1.00)$ & $1.00(0.70-1.00)$ \\
\hline & Yes & 337 & -0.51 & -0.0698 & & & & & $0.70(0.50-0.80)$ & $0.60(0.50-0.80)$ \\
\hline \multirow{2}{*}{$\begin{array}{l}\text { Other } \\
\text { retinopathy }\end{array}$} & No & 1129 & -0.23 & -0.0239 & 0.32 & 0.87 & 0.18 & 0.93 & $1.00(0.80-1.00)$ & $0.80(0.67-1.00)$ \\
\hline & Yes & 112 & -0.29 & -0.0370 & & & & & $0.80(0.67-1.00)$ & $0.67(0.50-1.00)$ \\
\hline \multirow{3}{*}{$\begin{array}{l}\text { Eye pressure } \\
(\mathrm{mmHg})\end{array}$} & $<16$ & 369 & -0.25 & -0.0215 & 0.58 & 0.64 & 0.031 & 0.045 & $1.00(0.70-1.00)$ & $0.90(0.67-1.00)$ \\
\hline & $16-<20$ & 592 & -0.22 & -0.0234 & & & & & $1.00(0.80-1.00)$ & $0.80(0.67-1.00)$ \\
\hline & $\geq 20$ & 151 & -0.28 & -0.0441 & & & & & $1.00(0.75-1.00)$ & $0.80(0.60-1.00)$ \\
\hline
\end{tabular}

Visual acuity is given as logMAR and decimal acuity. Estimates of logMAR are from a mixed model using all available measurements of visual acuity which come from the best seeing eye in all cases. $p$ values are from Wald tests. Median values (interquartile range) of decimal acuity are from the first and the last eye examination.

${ }^{a}$ Testing whether the mean of the level of visual acuity at diabetes diagnosis is different for the two or three levels of the stratification variable.

${ }^{\mathrm{b}}$ Testing whether the mean yearly change of visual acuity after diabetes diagnosis is different for the two or three levels of the stratification variable, given the level of the visual acuity at diabetes diagnosis. This test focuses on differences in slope between the linear curves describing changes in visual acuity.

${ }^{\mathrm{C}} \mathrm{HbA}_{1 \mathrm{c}}$ measured within 90 days of diabetes diagnosis. Reference range: 5.4-7.4.

(1.5\%) of 1241 patients. 13 had normal visual acuity and 5 had impaired vision.

\section{Change in visual acuity}

Among the 807 surviving and re-examined patients, visual acuity generally deteriorated (Table 3 ). The prevalence (95\% confidence interval) of blindness and moderate visual impairment was 2.4 (1.3-3.4) \% and 6.7 (5.0-8.4) \%, respectively, 5.6 (5.0-6.3) years (median, IQR) after the first eye examination. The 18 new-blind patients in Table 3 represent an incidence (95\% confidence interval) of blindness of 40.2 (25.3-63.8) per 10,000 patient-years. Of 18 new-blind patients, 14 were over 70 years. The incidence of moderately impaired vision or worse was 142.3 (110.5$183.2)$ per 10,000 patient-years among patients with normal sight at diagnosis. Of the 25 patients in Table 3 with moderate visual impairment at baseline, 14 had normal visual acuity 6 years later. Of these 14 patients, 7 had had a cataract operation since the baseline examination and one had had a retinal laser treatment. 
Table 2 Visual acuity at diabetes diagnosis according to age, sex, retinopathy, and cataract

\begin{tabular}{|c|c|c|c|c|}
\hline & \multicolumn{3}{|c|}{ Visual acuity $^{\text {a }}$} & \multirow[b]{2}{*}{ All patients } \\
\hline & Normal & $\begin{array}{l}\text { Moderately } \\
\text { impaired }\end{array}$ & Blind & \\
\hline & $n=1,163$ & $n=67$ & $n=11$ & $n=1,241$ \\
\hline \multicolumn{5}{|l|}{ Age (years) } \\
\hline $40-<60$ & $429(36.9)$ & $1(1.5)$ & $0(0)$ & $430(34.7)$ \\
\hline $60-<70$ & $366(31.5)$ & $10(14.9)$ & $1(9.1)$ & $377(30.4)$ \\
\hline $70+$ & $368(31.6)$ & $56(83.6)$ & $10(90.9)$ & $434(35.0)$ \\
\hline \multicolumn{5}{|l|}{ Sex } \\
\hline Male & $622(53.5)$ & $28(41.8)$ & $3(27.3)$ & $653(52.6)$ \\
\hline Female & $541(46.5)$ & $39(58.2)$ & $8(72.7)$ & $588(47.4)$ \\
\hline \multicolumn{5}{|l|}{ Retinopathy ${ }^{\mathrm{a}}$} \\
\hline \multicolumn{5}{|l|}{ Diabetic retinopathy } \\
\hline Microaneurysms only & $13(1.1)$ & $0(0)$ & $1(9.1)$ & $14(1.1)$ \\
\hline Further diabetic retinopathy & $34(2.9)$ & $2(3.0)$ & $2(18.2)$ & $38(3.1)$ \\
\hline Age-related macular degeneration, AMD & $117(10.1)$ & $30(44.8)$ & $7(63.6)$ & $154(12.4)$ \\
\hline Other & $104(8.9)$ & $7(10.5)$ & $1(9.1)$ & $112(9.0)$ \\
\hline No retinopathy & $908(78.3)$ & $32(47.8)$ & $1(9.1)$ & $941(76.0)$ \\
\hline Cataract $^{a}$ & $278(24.2)$ & $55(82.1)$ & $4(36.4)$ & $337(27.5)$ \\
\hline \multicolumn{5}{|l|}{$\begin{array}{l}\text { Ophthalmologist's indication of } \\
\text { reason for visual impairment }{ }^{a, b}\end{array}$} \\
\hline Retinopathy of all sorts & $76(6.5)$ & $13(19.4)$ & $5(45.4)$ & $94(7.6)$ \\
\hline Cataract & $171(14.7)$ & $25(37.3)$ & $1(9.1)$ & $197(15.9)$ \\
\hline Other causes & $42(3.6)$ & $6(9.0)$ & $2(18.2)$ & $50(4.0)$ \\
\hline Combination of causes & $45(3.9)$ & $20(29.9)$ & $2(18.2)$ & $67(5.4)$ \\
\hline None & $829(71.3)$ & $3(4.5)$ & $1(9.1)$ & $833(67.1)$ \\
\hline
\end{tabular}

Data are numbers (column-\%).

${ }^{a}$ Refers to best seeing eye.

${ }^{\mathrm{b}}$ The ophthalmologists answered this question even for patients who were mildly visually impaired although their visual acuity was normal per definition, i.e. $\geq 0.5$.

During the 6 years of follow up, DR had appeared in $11.7 \%(90 / 770)$ of patients without DR at diagnosis (Table 4). At 6-year follow up, DR and AMD were also relatively more common among the visually impaired (Table 5). The influence of eye complications at

Table 3 Changes in visual acuity from diabetes diagnosis until 6-year follow up

\begin{tabular}{lllll}
\hline $\begin{array}{l}\text { Visual acuity at } \\
\text { diagnosis }^{\mathbf{a}}\end{array}$ & \multicolumn{3}{l}{$\begin{array}{l}\text { Visual acuity at 6-year } \\
\text { follow up }^{\mathbf{a}}\end{array}$} & \multicolumn{2}{l}{} & \\
\cline { 2 - 5 } & Normal & $\begin{array}{l}\text { Moderately } \\
\text { impaired }\end{array}$ & Blind & Total \\
\hline Normal & $720(92.3)$ & $48(6.2)$ & $12(1.5)$ & 780 \\
Moderately impaired & $14(56.0)$ & $5(20.0)$ & $6(24.0)$ & $25(3.1)$ \\
Blind & $0(0)$ & $1(50.0)$ & $1(50.0)$ & $2(0.2)$ \\
Total & $734(90.9)$ & $54(6.7)$ & $19(2.4)$ & $807(100)$ \\
\hline
\end{tabular}

Data are numbers (row- and column-\%).

${ }^{\mathrm{a}}$ Refers to best seeing eye at diabetes diagnosis and 6-year follow up, respectively. diagnosis on change in visual acuity over 6 years was investigated in linear mixed models (Table 1). The 112 patients in Table 1 with "other retinopathy" presented with133 retinal pathologies other than DR and AMD: hypertensive retinopathy $(\mathrm{n}=43)$, retinal vasosclerosis (41), drusen (18) and other retinopathy (31). DR ( $\mathrm{n}=$ 52), AMD (154), and cataract (337) were associated with the level of visual acuity and/or its annual change also in age- and sex-adjusted analyses. These effects are illustrated with median decimal acuity values in Figure 2.

Similarly, some socio-demographic, clinical, biochemical, and behavioural variables affected the level of visual acuity and/or its annual change over 6 years even when the effect of age and sex was considered (Table 1). All significant effects, except that of self-rated health and triglycerides, are illustrated in Figure 2. The effect of triglycerides was not picked up by the median values. This is because the median figures in Figure 2 emphasize typical developments in visual acuity, while the result of 
the statistical analysis is necessarily based on $\log$ MAR values and expressed as means which are greatly affected by patients with very poor eye sight (Table 1 ).

When the changes in visual acuity were modelled as piecewise linear models with a breakpoint 3 years after diagnosis, the annual change in logMAR before $(0.0268$, $\mathrm{p}<0.0001)$ and after this point $(0.0231, \mathrm{p}=0.0008)$ was similar $(\mathrm{p}=0.99$ for the significance test of the time$\operatorname{logMAR}$ interaction). Accordingly, visual loss during the first years after diabetes diagnosis did not seem to be greater than later on, which supports the underlying assumption in Figure 2 that visual loss follows a linear course.

Adding the identification number of the eye doctors as a random effect to the models in Table 1 did not change the associations with developments in visual acuity. Therefore, it is unlikely that our results are affected by the fact that some patients were examined by the same eye doctor at baseline and follow up, while others were not.

\section{Discussion}

In a population-based cohort of patients newly diagnosed with clinical, often symptomatic type 2 diabetes, $6.3 \%$ were visually impaired. Among those patients with reduced sight, $76 \%$ had cataract and $58 \%$ retinopathy, usually AMD, although many of these eye pathologies are not closely related to diabetes and metabolic control. During the first 6 years after diabetes diagnosis, the incidence of blindness was relatively high, 40 per 10,000 person-years. The baseline predictors of both the level of and speed of progressive visual impairment after diagnosis were AMD, cataract, age at diagnosis, and living alone. The level of visual acuity over 6 years was lower in patients who had a low self-rated health or a sedentary life-style. The rate of the 6-year visual loss increased if the patient had DR or high fasting triglycerides at diabetes diagnosis.

\section{Strengths and limitations of the study}

Our patients are likely to be representative of Danish patients with newly diagnosed, clinical, often symptomatic diabetes in this age group because of the welldefined background population in each general practice, the unchanged inclusion activity during the inclusion period, the small number of primary exclusions, and the acceptable participation in the eye examinations [24]. Furthermore, data from studies including patients with known diabetes may be misleading because of selective survival of patients with a more favourable risk factor profile [29]. Our results, however, cannot be generalised to all countries because of marked disparities in causes of reduced vision across the world [8].

Our patient sample was established in the early 90's and since then both surgical treatment of eye pathologies and pharmacological treatment of hyperglycaemia, hypertension and dyslipidaemia have been intensified, as has screening for diabetes. These initiatives to improve diabetes care and to identify patients earlier in the natural history of diabetes have probably decreased the variability of measures of treatment quality, so if a similar follow up study were to be made today, the associations between variables would perhaps be harder to identify. There is, however, no reason to suppose that the causal patterns underlying the associations that we have identified would be substantially different.

By presenting results from the best seeing eye only, we are underestimating the prevalence of eye pathologies in this patient group. This is because our main purpose is to estimate the change in visual acuity and its predictors, and eye pathologies are important in this study only insofar they are predictors of visual loss. In most studies the main pathology in the better eye is considered the cause of visual impairment [6,7]. We analysed predictors of vision impairment prospectively. Furthermore, to improve statistical strength we chose to analyse visual acuity as a continuous outcome.

Our ability to detect a true clinical change in visual acuity is inversely related to the measurement error, i.e. the test-retest variability, of the test used [30,31]. Unlike logMAR charts, the Snellen chart, which we had to rely on in this nationwide primary care study, has a large-scale increment resulting in a relatively high measurement error. Another factor contributing to the testretest variability was differing routines for taking account of visual field loss [32]. The lower precision of the outcome, visual acuity, will however only tend to lessen the strength of the association between the outcome and a predictor.

On the other hand, the true incidence of vision impairment may be underestimated if those patients who missed an eye examination experienced a relatively rapidly declining vision. However there is no reason to suppose that the possible imprecision and error introduced were associated with any of the possible predictors of visual loss that were examined. Comparison of visual acuity between studies, even when categorised, is in any case not feasible [31], and our prevalence and incidence figures for visual impairment should be interpreted in this light.

Almost all Danish ophthalmologists contributed to the study increasing the inter-rater variability, and their screening by funduscopy may have overlooked $10-40 \%$ of sight-threatening eye disease [33]. Such measurement errors in the predictor variables will tend to reduce a true association between e.g. an eye pathology and the outcome, i.e. visual acuity, but it does not invalidate the associations that we actually find. It can be assumed 
Table 4 Prevalence of diabetic retinopathy at diabetes diagnosis and at 6-year follow up

\begin{tabular}{|c|c|c|c|c|c|c|}
\hline \multirow{3}{*}{$\begin{array}{l}\text { Diabetic retinopathy at } \\
\text { diabetes diagnosis }^{\mathrm{a}}\end{array}$} & \multicolumn{5}{|c|}{ Diabetic retinopathy at 6-year follow up ${ }^{a}$} & \multirow[b]{3}{*}{ Total } \\
\hline & \multirow{2}{*}{$\begin{array}{l}\text { No diabetic } \\
\text { retinopathy }\end{array}$} & \multicolumn{3}{|c|}{ Background retinopathy } & \multirow[b]{2}{*}{$\begin{array}{l}\text { Proliferative } \\
\text { retinopathy }\end{array}$} & \\
\hline & & $\begin{array}{l}\text { Microaneurysms } \\
\text { only }\end{array}$ & $\begin{array}{l}\text { Without } \\
\text { maculopathy }\end{array}$ & $\begin{array}{l}\text { With } \\
\text { maculopathy }\end{array}$ & & \\
\hline No diabetic retinopathy & $680(88.3)$ & $46(6.0)$ & $32(4.2)$ & $10(1.3)$ & $2(0.3)$ & $770(96.0)$ \\
\hline \multicolumn{7}{|l|}{ Background retinopathy } \\
\hline Microaneurysms only & $2(20.0)$ & $3(30.0)$ & $4(40.0)$ & $1(10.0)$ & $0(0)$ & $10(1.2)$ \\
\hline Without maculopathy & $8(47.1)$ & $1(5.9)$ & $3(17.6)$ & $1(5.9)$ & $4(23.5)$ & $17(2.1)$ \\
\hline With maculopathy & $0(0)$ & $1(20.0)$ & $0(0)$ & $3(60.0)$ & $1(20.0)$ & $5(0.6)$ \\
\hline Proliferative retinopathy & $0(0)$ & $0(0)$ & $0(0)$ & $0(0)$ & $0(0)$ & $0(0)$ \\
\hline Total & $690(86.0)$ & $51(6.4)$ & $39(4.9)$ & $15(1.9)$ & $7(0.9)$ & $802(100)$ \\
\hline
\end{tabular}

Data are numbers (row- and column-\%).

${ }^{a}$ Refers to the eye with the most pronounced diabetic retinopathy at diabetes diagnosis and 6-year follow up, respectively.

${ }^{\mathrm{b}}$ Among these, 2 patients with new vessels on the optic disc.

that the detection rate for eye disease was higher in patients with low visual acuity. This may have biased the cross-sectional associations between eye disease and visual acuity in Table 2 and 5, but it does not to the same extent compromise the estimation of the predictive power of the eye pathologies at diagnosis for the change in visual acuity during the following 6 years. Therefore, our non-standardized estimation of eye pathologies at diabetes diagnosis only diminishes our ability to detect an effect of these variables on changes in visual acuity.

Table 5 Visual acuity and retinopathy 6 years after diabetes diagnosis

\begin{tabular}{|c|c|c|c|c|}
\hline \multirow[b]{3}{*}{ Retinopathy $^{a}$} & \multicolumn{3}{|c|}{ Visual acuity $^{a}$} & \multirow{3}{*}{$\begin{array}{l}\text { All } \\
\text { patients } \\
n=804\end{array}$} \\
\hline & Normal & $\begin{array}{l}\text { Moderately } \\
\text { impaired }\end{array}$ & Blind & \\
\hline & $n=734$ & $n=54$ & $n=16$ & \\
\hline \multicolumn{5}{|l|}{ Diabetic retinopathy } \\
\hline $\begin{array}{l}\text { Microaneurysms } \\
\text { only }\end{array}$ & $46(6.3)$ & $5(9.3)$ & $0(0)$ & $51(6.3)$ \\
\hline $\begin{array}{l}\text { Further diabetic } \\
\text { retinopathy }{ }^{b}\end{array}$ & $48(6.5)$ & $10(18.5)$ & $3(18.8)$ & $61(7.6)$ \\
\hline $\begin{array}{l}\text { Age-related macular } \\
\text { degeneration, AMD }\end{array}$ & $65(8.9)$ & $20(37.0)$ & $11(57.9)$ & 96 (11.9) \\
\hline Other retinopathy & $44(6.0)$ & $0(0)$ & $2(10.5)$ & $46(5.7)$ \\
\hline No retinopathy & $541(73.7)$ & $23(42.6)$ & $1(6.3)$ & $565(70.3)$ \\
\hline
\end{tabular}

\section{Comparison with existing literature}

\section{Predictors of 6-year visual loss}

In Table 2 and in most studies [3-9,34-36] the main eye pathology is assigned as the cause of visual impairment. We analysed prospectively 26 possible ocular and nonocular predictors of vision loss (Table 1). It is striking that, besides age and triglycerides, only DR, AMD, and cataract, many of which are surgically modifiable, were associated with declining vision over 6 years. This was observed even though the measurement error in the estimation of these eye pathologies is considered to be greater than for many of the other possible predictors. In follow up studies, the association between baseline DR or AMD and later impaired vision is well documented [14-16,19], and AMD may cause deterioration in visual acuity earlier in diabetic patients than in nondiabetic people though the prevalence of AMD does not seem to differ markedly between the two groups [17]. Furthermore, diabetic subjects have a 2 to 4 times greater risk of developing cataract than non-diabetic people [18].

As our way of collecting information about glaucoma may underestimate the true prevalence of glaucoma in our patients, we were not able to analyse the predictive effect of glaucoma for visual loss. The possible importance of eye pressure for the change in eye sight over 6 years is, however, indicated by the non-significant tendency reported in Table 1.

Only a few prospective studies have assessed nonocular predictors of visual loss other than age and sex $[14,16,19-23]$. The only study including more than a few possible predictors used a subjective measure of visual dysfunction [14], while the most comprehensive study until now using measured visual acuity examined the 


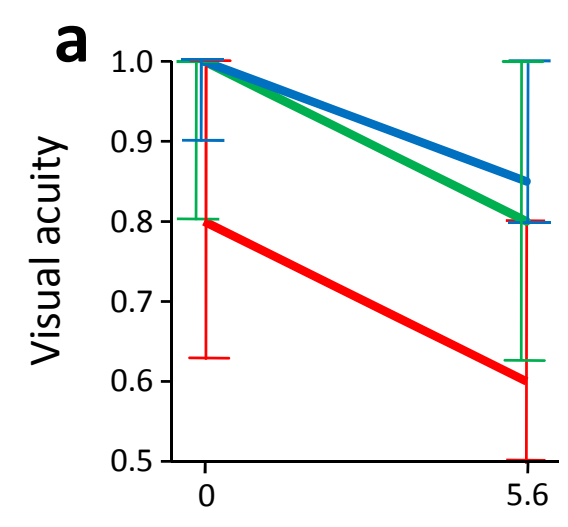

Years since diabetes diagnosis

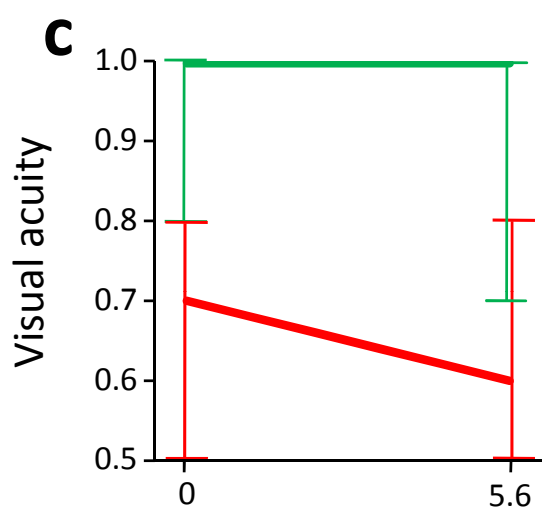

Years since diabetes diagnosis

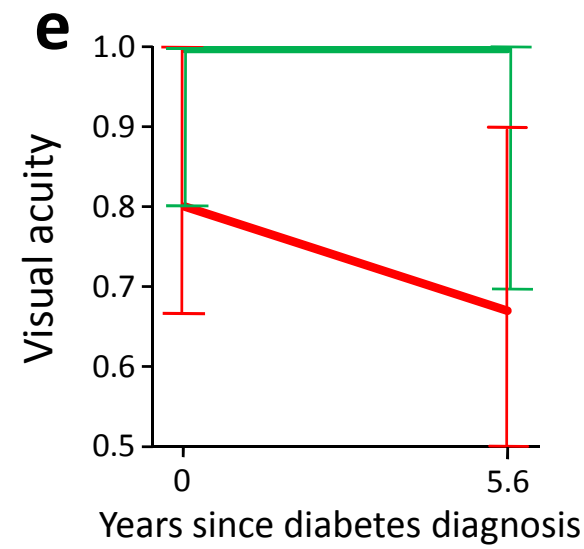

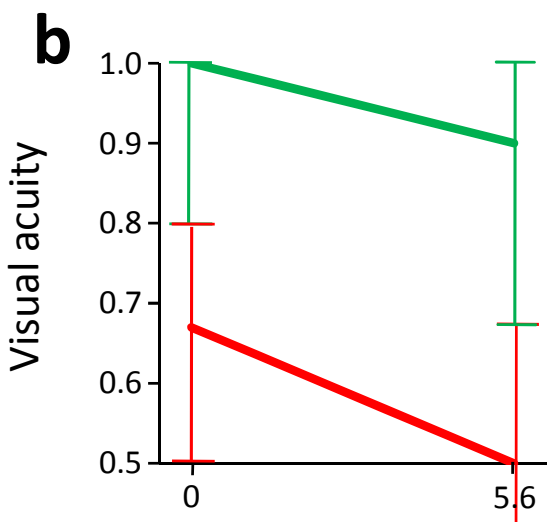

Years since diabetes diagnosis

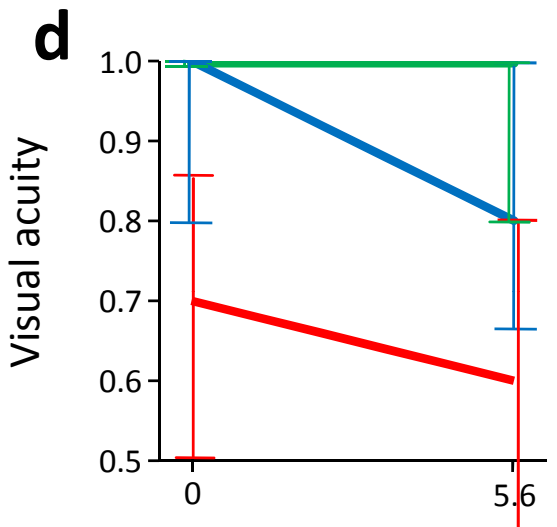

Years since diabetes diagnosis

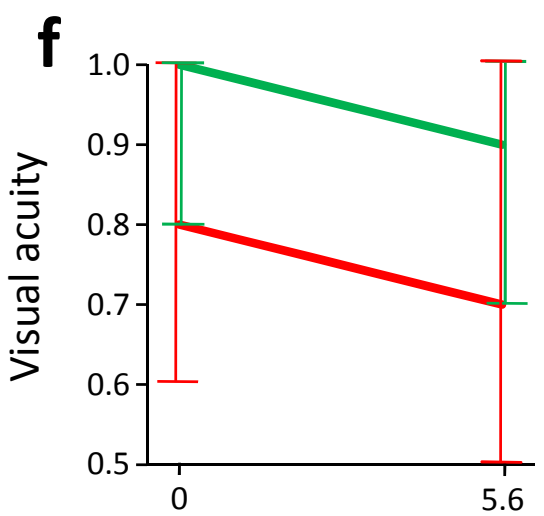

Years since diabetes diagnosis

Figure 2 Vision loss during the first 6 years after diabetes diagnosis according to all statistically significant predictors at diagnosis except fasting triglycerides and self-rated health. The curves are defined by medians of decimal acuity (interquartile ranges) at diagnosis and 6 years later. a Diabetic retinopathy: no retinopathy (green); microaneurysms only (blue); further retinopathy (red). b Age-related macular degeneration: no (green); yes (red). c Cataract: no (green); yes (red). d Age, years: 40- < 60 (green); 60- < 70 (blue); $\geq 70$ (red). e Living alone: no (green); yes (red). f Physical activity: moderate/high (green); low (red). 
effect of HbA1c, blood pressures, proteinuria and smoking as well as age and sex [19]. Among many candidate predictors we found only relatively high age, living alone and high triglycerides to be associated with worsening of visual acuity over 6 years, while high age, living alone, low self-rated health, and low level of physical activity were associated with a low level of visual acuity. Presumably the three last-mentioned relations are cases of reverse causation where poor vision affects living conditions. Marital status has similarly been found to predict vision loss in men with older-onset diabetes [20], but the association was reversed in a study of patients with advanced DR [21].

In UKPDS the incidence of visual impairment was slightly lower in the tight vs. the less tight blood pressure control group [22]. Similarly, blood pressure, $\mathrm{HbA} 1 \mathrm{c}$ and proteinuria have been shown to be indicative of visual loss in follow up studies $[16,19,23]$, but none of these non-ocular patient variables was associated with visual loss in the present study. This could be due to measurement error and above all regression dilution bias [37], which is particularly relevant for biochemical and clinical variables in the dysmetabolic state of newly diagnosed clinical diabetes. In studies including patients with known diabetes $[16,19,23]$, the measured risk factor levels are supposedly closer to an average steady state level, a kind of set point which is typical for the patient in question. In the present study, however, high level of triglycerides, which has been identified as a risk factor for proliferative DR [38], was a significant predictor of declining vision. In line with this finding, the FIELD study showed a promising reduction in the need for laser treatment for DR after treatment with fenofibrate but this did not affect worsening of visual acuity [39]. It is possible that DR mediates the effect of triglycerides on visual acuity in a slow, progressive pathophysiological process. The strong counter-intuitive inverse univariate relation between smoking and visual acuity disappeared after age and sex adjustment (Table 1) while a similar association between smoking and DR persisted after adjustment in UKPDS [40].

\section{Prevalence and incidence of blindness and moderate visual impairment}

In population-based studies of patients with known type 2 diabetes the prevalence of blindness is between $1 \%$ and $3 \%[6,7,34,41-43]$, lowest in populations offered regular eye screening. The prevalence increases markedly with diabetes duration $[14,23,42,43]$, in the present study from $0.9 \%$ at diagnosis to $2.4 \%$ six years later (Table 3).

With the gradual implementation of systematic eye screening the incidence of blindness among diabetic patients has declined [44]. In the Nordic countries the incidence per 10,000 person-years has declined from the range of $200-500[15,45]$ in the early 1980 s to more recent figures of about $15[23,46]$ among persons with known type 2 diabetes. Our patient sample included many old patients and both the prevalence $[2,4,41]$ and the incidence $[14,16,19]$ of visual impairment increases curvelinearly with age as in the background population $[2,36,47]$. This may partially explain why the incidence rate was as high as 40 per 10,000 person-years in the present study.

In studies of patients with known type 2 diabetes, which are to some extent population-based, the prevalence of $7-11 \%$ for moderate visual impairment $[22,34,42,43]$ is similar to the $6.7 \%$ observed 6 years after diagnosis in the present study. Of the 54 patients with moderate visual impairment at this point, however, 48 had normal vision at diagnosis, but the prevalence rate increased only modestly because of the over-mortality of patients with low vision [48].

\section{Implications for clinical practice and future research}

It is evident that even in Denmark where patients can refer themselves to free eye examinations, treatable eye pathologies such as DR and cataract predict further visual loss. Our results underline the importance of eliminating barriers to efficient eye care by facilitating access to eye examination, increasing the understanding of patients and primary care practitioners of the need for regular screening and early surgical treatment, and, in some countries, addressing patients' financial burdens [49]. It should be easy to motivate a thoroughly informed patient to have regular eye examinations as many patients fear visual loss as the worst consequence of diabetes $[10,13]$. The fact that almost all Danish primary care eye doctors participated in the present study demonstrates the commitment of ophthalmologists to preventive diabetes care.

In primary care, future intervention studies to reduce visual impairment in patients with diabetes may be designed primarily to overcome barriers to effective eye care, and such trials should preferably distinguish between the effect on visual acuity of changes in biological age on retina and of the interventions targeting treatable eye pathologies.

\section{Conclusions}

To conclude, severely reduced sight is a very real challenge for patients with newly diagnosed clinical, often symptomatic type 2 diabetes. During the first 6 years after diabetes diagnosis visual acuity deteriorates considerably, and this visual loss depends primarily on age and the presence of AMD, DR and cataract at diagnosis. Patients newly diagnosed with clinical type 2 diabetes should be made aware that there is an inevitable agerelated decline in sight but that further vision loss 
associated with diabetes is largely preventable through diligent ophthalmological follow up and surgical intervention.

\section{Acknowledgements}

We are grateful to the patients, ophthalmologists and general practitioners who participated in the study and to Lise Bergsøe for her expert technical assistance. Major funding for this study was received from The Danish Medical Research Council, The Danish Research Foundation for General Practice, The Health Insurance Foundation, The Danish Ministry of Health, Novo Nordisk Farmaka Denmark Ltd., and The Pharmacy Foundation.

\section{Author details}

${ }^{1}$ The Research Unit for General Practice and Section of General Practice, Department of Public Health, University of Copenhagen, Copenhagen, Denmark. ${ }^{2}$ University Eye Clinic, Rigshospitalet, Copenhagen, Denmark.

\section{Authors' contributions}

NDFO conceived of and designed the study, carried out data collection, and drafted the manuscript. NDFO and NVN designed and coded the eye questionnaires. VS did the statistical analyses. NDFO, VS, GJA and NVN participated in the interpretation of data and in the revisions of the manuscript. All authors have read and approved the final manuscript.

\section{Competing interests}

The authors declare that they have no competing interests.

Received: 19 August 2010 Accepted: 4 February 2011

Published: 4 February 2011

\section{References}

1. Hayward LM, Burden ML, Burden AC, Blackledge H, Raymond NT, Botha JL, Karwatowski WS, Duke T, Chang YF: What is the prevalence of visual impairment in the general and diabetic populations: are there ethnic and gender differences? Diabet Med 2002, 19:27-34.

2. Zhang X, Gregg EW, Cheng YJ, Thompson TJ, Geiss LS, Duenas MR, Saaddine JB: Diabetes mellitus and visual impairment: national health and nutrition examination survey, 1999-2004. Arch Ophthalmol 2008, 126:1421-1427.

3. Rosenberg T, Klie F: Current trends in newly registered blindness in Denmark. Acta Ophthalmol Scand 1996, 74:395-398.

4. Ghafour IM, Allan D, Foulds WS: Common causes of blindness and visual handicap in the west of Scotland. Br J Ophthalmol 1983, 67:209-213.

5. Bamashmus MA, Mathaga B, Dutton GN: Causes of blindness and visual impairment in the West of Scotland. Eye 2004, 18:257-261.

6. Jeppesen P, Bek T: The occurrence and causes of registered blindness in diabetes patients in Arhus County, Denmark. Acta Ophthalmol Scand 2004, 82:526-530.

7. Prasad S, Kamath GG, Jones K, Clearkin LG, Phillips RP: Prevalence of blindness and visual impairment in a population of people with diabetes. Eye 2001, 15:640-643.

8. Resnikoff S, Pascolini D, Etya'ale D, Kocur I, Pararajasegaram R, Pokharel GP, Mariotti SP: Global data on visual impairment in the year 2002. Bull World Health Organ 2004, 82:844-851.

9. Buch H, Vinding T, La CM, Nielsen NV: The prevalence and causes of bilateral and unilateral blindness in an elderly urban Danish population. The Copenhagen City Eye Study. Acta Ophthalmol Scand 2001, 79:441-449.

10. Coyne KS, Margolis MK, Kennedy-Martin T, Baker TM, Klein R, Paul MD, Revicki DA: The impact of diabetic retinopathy: perspectives from patient focus groups. Fam Pract 2004, 21:447-453.

11. Woodcock A, Bradley C, Plowright R, Ffytche T, Kennedy-Martin T, Hirsch A: The influence of diabetic retinopathy on quality of life Interviews to guide the design of a condition-specific, individualised questionnaire: the RetDQoL. Patient Educ Couns 2004, 53:365-383.

12. Brown MM, Brown GC, Sharma S, Shah G: Utility values and diabetic retinopathy. Am J Ophthalmol 1999, 128:324-330.

13. Luckie R, Leese G, McAlpine R, MacEwen CJ, Baines PS, Morris AD, Ellis JD: Fear of visual loss in patients with diabetes: results of the Prevalence of
Diabetic Eye Disease in Tayside, Scotland (P-DETS) study. Diabet Med 2007, 24:1086-1092.

14. Miki E, Lu M, Lee ET, Keen H, Bennett PH, Russell D: The incidence of visual impairment and its determinants in the WHO multinational study of vascular disease in diabetes. Diabetologia 2001, 44:S31-S36.

15. Nielsen NV: Diabetic retinopathy II. The course of retinopathy in diabetics treated with oral hypoglycaemic agents and diet regime alone. A one year epidemiological cohort study of diabetes mellitus. The Island of Falster, Denmark. Acta Ophthalmol 1984, 62:266-273.

16. Moss SE, Klein R, Klein BEK: The Incidence of Vision Loss in A Diabetic Population. Ophthalmology 1988, 95:1340-1348.

17. Voutilainen-Kaunisto RM, Terasvirta ME, Uusitupa MIJ, Niskanen LK: Agerelated macular degeneration in newly diagnosed type 2 diabetic patients and control subjects - A 10-year follow-up on evolution, risk factors, and prognostic significance. Diabetes Care 2000, 23:1672-1678.

18. Klein BEK, Klein R, Moss SE: Incidence of Cataract-Surgery in the Wisconsin Epidemiologic-Study of Diabetic-Retinopathy. Am J Ophthalmol 1995, 119:295-300.

19. Moss SE, Klein R, Klein BE: Ten-year incidence of visual loss in a diabetic population. Ophthalmology 1994, 101:1061-1070.

20. Klein R, Klein BE, Jensen SC, Moss SE: The relation of socioeconomic factors to the incidence of proliferative diabetic retinopathy and loss of vision. Ophthalmology 1994, 101:68-76.

21. Rand LI, Prudhomme GJ, Ederer F, Canner PL: Factors Influencing the Development of Visual-Loss in Advanced Diabetic-Retinopathy Diabetic-Retinopathy Study (Drs) Report No-10. Invest Ophthalmol Vis Sci 1985, 26:983-991.

22. Matthews DR, Stratton IM, Aldington SJ, Holman RR, Kohner EM: UKPDS 69. Risks of progression of retinopathy and vision loss related to tight blood pressure control in type 2 diabetes mellitus. Arch Ophthalmol 2004, 122:1631-1640.

23. Henricsson $M$, Tyrberg $M$, Heijl A, Janzon L: Incidence of blindness and visual impairment in diabetic patients participating in an ophthalmological control and screening programme. Acta Ophthalmol Scand 1996, 74:533-538.

24. Olivarius NdF, Beck-Nielsen $\mathrm{H}$, Andreasen AH, Horder M, Pedersen PA: Randomised controlled trial of structured personal care of type 2 diabetes mellitus. BMJ 2001, 323:970-975.

25. Holladay JT: Visual acuity measurements. J Cataract Refract Surg 2004, 30:287-290.

26. Beatty OL, Ritchie CM, Bell PM, Hadden DR, Kennedy L, Atkinson AB: Microalbuminuria as identified by a spot morning urine specimen in non-insulin-treated diabetes: an eight-year follow-up study. Diabet Med 1995, 12:261-266.

27. Verbeke G, Molenberghs G: Linear Mixed Models for Longitudinal Data. Springer series in statistics New York: Springer; 2000.

28. Benjamini $Y$, Hochberg Y: Controlling the False Discovery Rate: a Practical and Powerful Approach to Multiple Testing. J Roy Stat Soc B 1995, 57:289-300.

29. Ballard DJ, Melton LJ: Sources of disparity in incidence and prevalence studies of diabetic retinopathy: influence of selective survival on risk factor assessment [letter]. Diabetes Care 1986, 9:313-315.

30. Rosser DA, Murdoch IE, Cousens SN: The effect of optical defocus on the test-retest variability of visual acuity measurements. Invest Ophthalmol Vis Sci 2004, 45:1076-1079.

31. Ricci F, Cedrone C, Cerulli L: Standardized measurement of visual acuity. Ophthalmic Epidemiol 1998, 5:41-53

32. Taylor HR, Livingston PM, Stanislavsky YL, McCarty CA: Visual impairment in Australia: distance visual acuity, near vision, and visual field findings of the Melbourne Visual Impairment Project. Am J Ophthalmol 1997, 123:328-337.

33. Harding SP, Broadbent DM, Neoh C, White MC, Vora J: Sensitivity and specificity of photography and direct ophthalmoscopy in screening for sight threatening eye disease: the Liverpool Diabetic Eye Study. BMJ 1995, 311:1131-1135.

34. Fong DS, Sharza M, Chen W, Paschal JF, Ariyasu RG, Lee PP: Vision loss among diabetics in a group model Health Maintenance Organization (HMO). Am J Ophthalmol 2002, 133:236-241.

35. Dwyer MS, Melton LJ III, Ballard DJ, Palumbo PJ, Trautmann JC, Chu CP: Incidence of diabetic retinopathy and blindness: a population-based study in Rochester, Minnesota. Diabetes Care 1985, 8:316-322. 
36. Attebo K, Mitchell P, Smith W: Visual acuity and the causes of visual loss in Australia - The blue mountains eye study. Ophthalmology 1996, 103:357-364

37. Smith GD, Phillips AN: Inflation in epidemiology: "The proof and measurement of association between two things" revisited. BMJ 1996, 312:1659-1661.

38. Davis MD, Fisher MR, Gangnon RE, Barton F, Aiello LM, Chew EY, Ferris FL, Knatterud GL: Risk factors for high-risk proliferative diabetic retinopathy and severe visual loss: Early treatment diabetic retinopathy study report \#18. Invest Ophthalmol Vis Sci 1998, 39:233-252.

39. Keech AC, Mitchell P, Summanen PA, O'Day J, Davis TM, Moffitt MS, Taskinen MR, Simes RJ, Tse D, Williamson E, et al: Effect of fenofibrate on the need for laser treatment for diabetic retinopathy (FIELD study): a randomised controlled trial. Lancet 2007, 370:1687-1697.

40. Stratton IM, Kohner EM, Aldington SJ, Turner RC, Holman RR, Manley SE, Matthews DR: UKPDS 50: risk factors for incidence and progression of retinopathy in Type II diabetes over 6 years from diagnosis. Diabetologia 2001, 44:156-163.

41. Klein R, Klein BE, Moss SE: Visual impairment in diabetes. Ophthalmology 1984, 91:1-9.

42. Idil A, Caliskan D, Ocaktan E: The prevalence of blindness and low vision in older onset diabetes mellitus and associated factors: a communitybased study. Eur J Ophthalmol 2004, 14:298-305.

43. Olafsdottir E, Andersson DK, Stefansson E: Visual acuity in a population with regular screening for type 2 diabetes mellitus and eye disease. Acto Ophthalmol Scand 2007, 85:40-45.

44. Trautner C, Haastert B, Giani G, Berger M: Incidence of blindness in southern Germany between 1990 and 1998. Diabetologia 2001, 44:147-150.

45. Voutilainen-Kaunisto RM, Terasvirta ME, Uasitupa MIJ, Niskanen LK: Occurrence and predictors of retinopathy and visual acuity in Type 2 diabetic patients and control subjects 10-year follow-up from the diagnosis. J Diab Comp 2001, 15:24-33.

46. Agardh E, Agardh CD, Hansson-Lundblad C: The five-year incidence of blindness after introducing a screening programme for early detection of treatable diabetic retinopathy. Diabet Med 1993, 10:555-559.

47. Klein R, Klein BEK, Lee KE, Cruickshanks KJ, Gangnon RE: Changes in visual acuity in a population over a 15-year period: The Beaver Dam Eye Study. Am J Ophthalmol 2006, 142:539-549.

48. Knudtson MD, Klein BEK, Klein R: Age-related eye disease, visual impairment, and survival - The Beaver Dam Eye Study. Arch Ophthalmol 2006, 124:243-249.

49. Hartnett ME, Key IJ, Loyacano NM, Horswell RL, DeSalvo KB: Perceived barriers to diabetic eye care - Qualitative study of patients and physicians. Arch Ophthalmol 2005, 123:387-391.

\section{Pre-publication history}

The pre-publication history for this paper can be accessed here: http://www.biomedcentral.com/1471-2458/11/80/prepub

doi:10.1186/1471-2458-11-80

Cite this article as: de Fine Olivarius et al:: Prevalence and progression of visual impairment in patients newly diagnosed with clinical type 2 diabetes: a 6-year follow up study. BMC Public Health 2011 11:80.

\section{Submit your next manuscript to BioMed Central and take full advantage of:}

- Convenient online submission

- Thorough peer review

- No space constraints or color figure charges

- Immediate publication on acceptance

- Inclusion in PubMed, CAS, Scopus and Google Scholar

- Research which is freely available for redistribution 